\title{
Hospital Pharmacy and Research
}

\author{
Christian Surber*, Carolina Pellanda, Katja Zimmermann, Manuela Endres, Verena Figueiredo, \\ Roland Werner, Herbert Plagge, and Ruth Leu Marseiler
}

\begin{abstract}
Hospital pharmacies play a crucial role in managing and/or accompanying the entire drug handling process, from the drug's origin all the way through the hospital system to the patient, and to promote rational and appropriate drug use. Drug preparation - still part of this process - has not disappeared from the fields of activity of hospital pharmacists. However, this activity is bound to many legal constraints and, therefore, remains reserved to a limited circle of hospitals that can offer such services. Based on a national license (Swissmedic), the Institute of Hospital Pharmacy of the University Hospital of Basel offers opportunities to provide drug preparations according to current guidelines. These preparations include patient-individualized drug preparations (compounding) to fulfil special needs in oncology, dermatology, paediatrics and other disciplines. Additionally this national license enables our institution to prepare drug preparations for clinical research. This is the basis of an important activity of our institute: to prepare clinical research samples for the pharmaceutical industry and for clinicians at the university hospital, to support industry independent research, and to conduct our own research in biopharmaceutics in collaboration with other national and international institutions. Against this background we report on our research support activities, and, in more detail, on our own research and development programs in biopharmaceutics: dermal and intranasal bioavailability.
\end{abstract}

Keywords: Clinical pharmacy · Cutaneous administration · Hospital pharmacy · Intranasal administration . Topical bioavailability

\section{Introduction}

The history of the origin of hospital pharmacies is closely related to the formation of hospital-like institutions (lazarettos) and hospitals, and in Europe they may be traced back to medieval times. The main focus of pharmacies or drug dispensaries has always been on preparing and distributing medicinal products. Preparing medicine was originally an empirical craftsman's work, influenced by the current mainstream of medicine. Hospitals were supplied with

${ }^{\star}$ Correspondence: Prof. Dr. phil. II C. Surber Institute of Hospital Pharmacy

University Hospital Basel

Spitalstrasse 26

$\mathrm{CH}-4031$, Base

Tel.: +4161265 2905

Fax: +4161265 8875

E-Mail: christian.surber@unibas.ch

www.spitalpharmazie-basel.ch medicinal products by either in-house institutions, or more often by local public pharmacies. With the beginning of the 19th century, the preparation of medicinal products became more science-based, and pharmaceutical industrialization was heralded. The activities of most hospital pharmacies have changed since then, and drug distribution as well as drug information for other healthcare professionals and for the patient became important tasks of hospital pharmacists. The present evolution in our healthcare system expects the hospital pharmacist to be an expert in managing and/or accompanying the entire drug handling process, from the drug's origin all the way through the hospital system to the patient, and to promote rational and appropriate drug use.

Along with this drug handling process, a relatively young field of research has evolved focusing on the entire medication process, including drug utilization and drug safety. Recently pharmacoeconomic questions have also reached this field. Despite this development, drug preparation has not completely disappeared from the fields of activity of hospital pharmacists. However, this activity is bound to many legal constraints; and, therefore, remains reserved to a limited circle of hospitals that can offer such services. Based on a national license (Swissmedic), the Institute of Hospital Pharmacy of the University Hospital of
Basel offers opportunities to provide drug preparations according to current guidelines (e.g. GMP; Good Manufacturing Practice). These preparations include patient-individualized drug preparations (compounding) to fulfil special needs in oncology, dermatology, paediatrics and other disciplines. Additionally this national license enables our institution to prepare drug preparations for clinical research. This is the basis of an important activity of our institution: to prepare clinical research samples for the pharmaceutical industry and for clinicians at the university hospital, as well as to support industry-independent research, and to conduct our own research in biopharmaceutics in collaboration with the Departments of Pharmaceutical Sciences and Forensic Chemistry and Toxicology of the University of Basel, the University Hospital Basel and other universities, e.g. Campus Charité Berlin, University of South Carolina, or University of California San Francisco.

Against this background we herein report on our research support activities and on our own research and development programs in biopharmaceutics.

\section{Research Support Activity}

To comply with the legal requirements for clinical trials, considerable and com- 
prehensive efforts have to be taken. These comprise - among many other aspects - an unbroken documentation of the origin and the quality of the clinical trial samples. We offer the pharmaceutical industry and the clinicians at our institution the preparation of clinical trial samples that meet the legal requirements, i.e. the required seamless documentation on the production and the quality of the clinical trial samples. The activities include the following: preparation of sterile and non-sterile products, keeping in stock or preparing ad hoc, labelling, randomizing, blinding, adequate storage and distribution. The storage of randomization codes - accessible $24 \mathrm{~h} / 365 \mathrm{~d}$ - and the support in administrative matters complete the research support activities. Typical examples are the filling of MEMS containers (Micro-Electro-Mechanical Systems) with a variety of drugs used in compliance research, or the preparation of capsules and injection/infusion solutions with ${ }^{13} \mathrm{C}$ labelled glucose used for investigating glucose metabolism in patients with the metabolic syndrome and familial forms of hypertrigyceridemia. Specialized in preparing small batches of clinical trial samples, we satisfy an important need of the pharmaceutical industry and clinicians at our hospital.

\section{Research and Development in Biopharmaceutics}

Our own research is focused on the investigation of the influence of pharmaceutical vehicles on the topical bioavailability of selected drug classes. The latter include corticosteroids and sunscreen agents for cutaneous application, and benzodiazepines for intranasal application. Standardization and validation of sampling techniques and analytical methods are also key activities in our remit. These activities form the prerequisite for investigating the influence of pharmaceutical vehicles and other aspects of daily (clinical) routine on the drug bioavailability.

Bioavailability assessment of topically applied drugs requires the differentiation between two types of bioavailability: local bioavailability (Section 3.1.), and systemic bioavailability (Section 3.2.). If a topically applied drug is meant to act locally, i.e. if the skin is the target site, then the local bioavailability is of primary interest. However, if a topically applied drug is meant to act systemically, then the systemic bioavailability is of primary interest. The determination of bioavailability is generally based on the determination of drug concentrations - either in blood (systemic bioavailability) or in a target site such as the skin (local bioavailability).

\subsection{Local Bioavailability after Topical Application}

\subsubsection{Determination of Local Bioavailability by Tape-Stripping}

Topical delivery can be defined as the application of a drug- or xenobiotic-containing formulation to the skin in order to directly treat a cutaneous disorder (e.g. acne), a cutaneous manifestation of a general disease (e.g. psoriasis), or to protect the skin from an environmental intrusion (e.g. sunlight, pesticides), with the intent of confining the pharmacological or protective effects within or at the surface of the skin. Corticosteroids, sunscreen agents as well as other drugs such as antimycotics or antivirals are typical examples where local bioavailability is of major interest, because the compound is meant to act locally.

A vast range of in vitro and in vivo methods for the assessment of the local bioavailability has been proposed. One of the most promising methods is the tape-stripping technique [1], which has been found useful in dermatological research for selectively, and at times, exhaustively removing the skin's outermost layer, the stratum corneum. Typically, an adhesive tape is pressed onto the skin and is subsequently abruptly removed. The tape application and removal procedure may be repeated up to 100 times (Fig. 1). The introduction of the tape stripping method to investigate the reservoir and the barrier function of the skin gave a significant expansion to experimental tools in skin research.

The general technique used to date involves topical dosing, followed by application and subsequent removal of adhesive tape strips from the skin; chemicals and corneocytes adhering to the tape strips

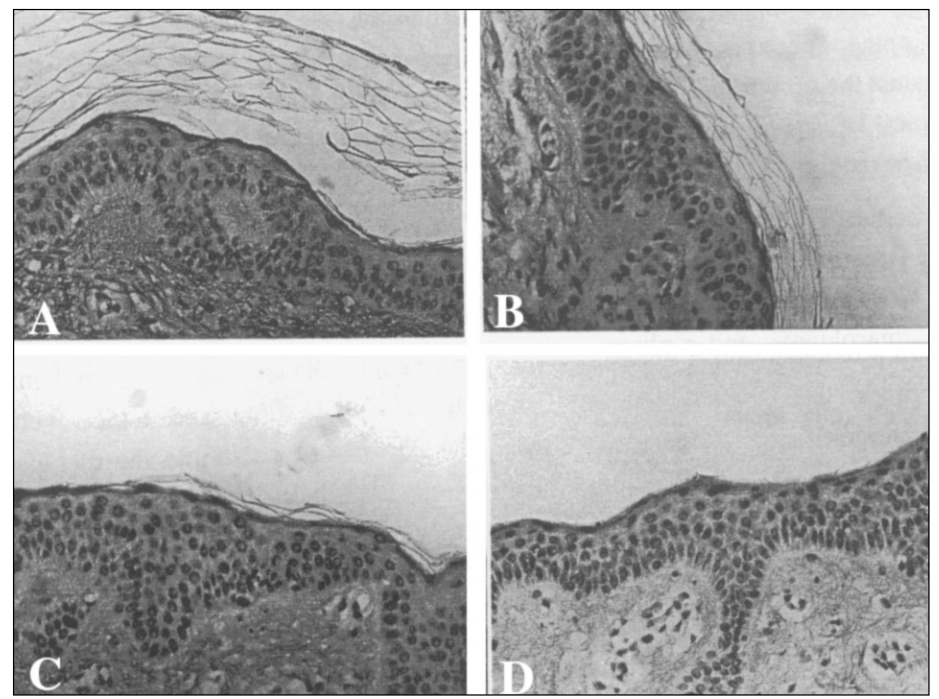

Fig. 1. Microscopic cross section of human skin with: A) intact stratum corneum; B) stratum corneum after stripping of 10 tapes; C) stratum corneum after stripping of 50 tapes; D) stratum corneum after stripping of 100 tapes [2] are thereby removed from the underlying tissue. The amount of chemical found on each strip is then related to the tape strip number, the mass of the corneocyte orthe protein content of tissue removed with each strip (Fig. 2). Some of the procedures are laborious and susceptible to numerous artefacts. Therefore standardization and simplification of the experimental workflow is crucial. Recently we researched a technique that allows, depending on the chemical properties of the permeant (e.g. sunscreen agents in this case), the simultaneous analysis of corneocyte density and xenobiotic abundance using a spectrophotometer, modified to monitor a rectangular beam of $1 \mathrm{~cm}^{2}$. This technique has distinct advantages in terms of facile sample handling procedures compared to previously described methods. The presumption that factors that improve percutaneous absorption also result in an increase of the drug reservoir in the stratum corneum, gave the tape stripping methodology one possible approach for selecting or comparing vehicles for topical drugs (Fig. 3).

Data from tape-stripping experiments can therefore be related to chemical penetration into skin, chemical permeation through the skin, and chemical elimination from the skin, as well as to pharmacodynamic and clinical parameters.

\subsubsection{Topical Triamcinolone Acetonide under Clinical Investigation}

In two clinical trials, the pharmacokinetic and pharmacodynamic profiles of triamcinolone acetonide (a model compound in our present research endeavour) formulations were determined. In a dermatopharmacokinetic investigation, the effect of clinically relevant aspects of dosing such as 


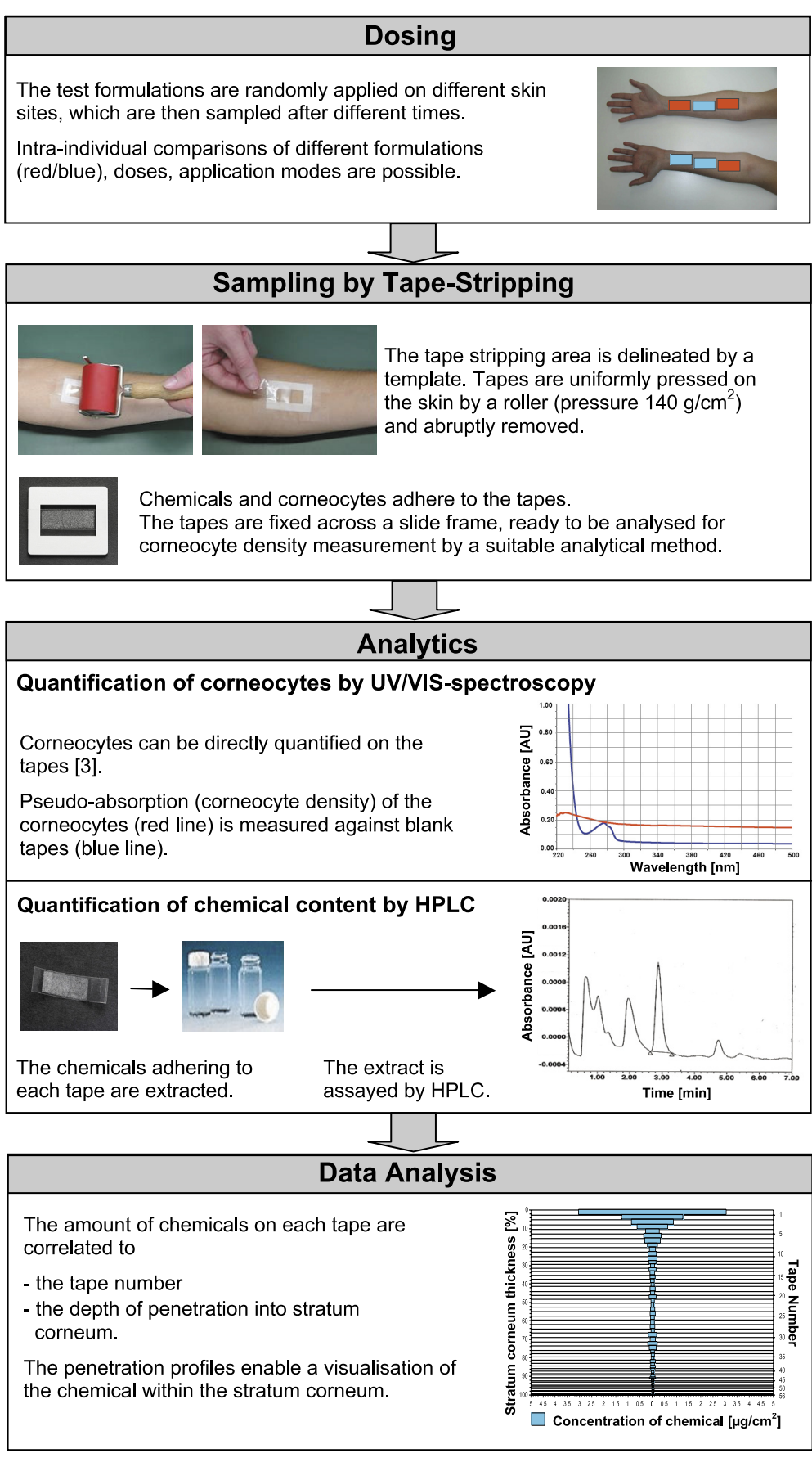

Fig. 2. Outline of a typical tape-stripping experiment. Skin site, type of tape used, pressure applied on the tape prior to removal, and applied vehicle are all factors influencing the amount of stratum corneum removed by each tape. Therefore the standardization of the technique is crucial. Chemical extraction from the tape is required when the absorption maximum is below $290 \mathrm{~nm}$ (e.g. corticosteroids), where as chemicals with an absorption maximum above $290 \mathrm{~nm}$ can be quantified simultaneously with the corneocyte density on the tape by UV/VIS spectroscopy (e.g. sunscreen agents).
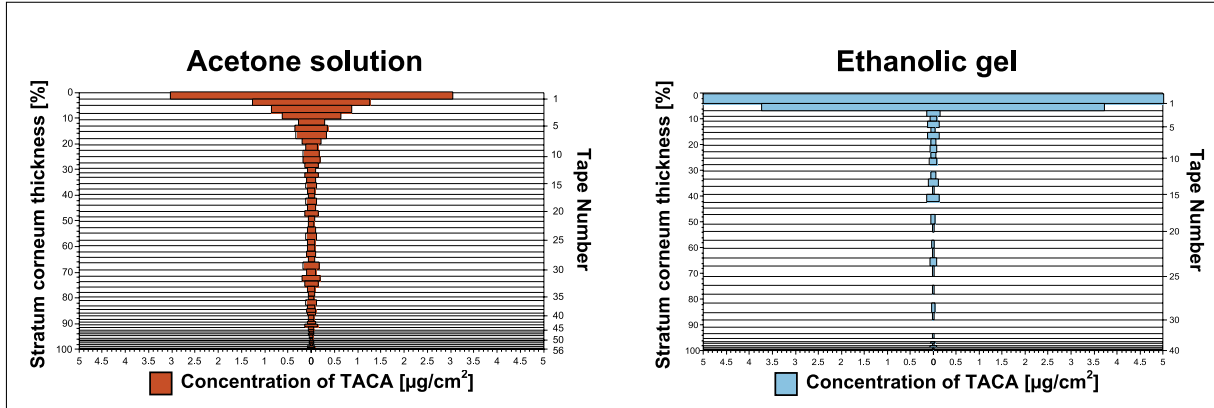

the effect of the applied dose (high vs. low), the application frequency (once daily vs. thrice daily), and occlusion were assessed by tape-stripping in human volunteers [4]. In a double blind, randomized, vehicle-controlled clinical trial, the effect of a low-dose triamcinolone acetonide formulation was assessed in patients with atopic dermatitis. The low-dose triamcinolone acetonide formulation was found to be effective in the treatment of mild to moderate atopic dermatitis [5]. The findings justify the use of much lower corticosteroid concentrations than typically marketed and used today, particularly in subacute and chronic eczema, and in children.

\subsection{Systemic Bioavailability after Topical Application}

3.2.1. Intranasal Application of Midazolam

Intranasal drug delivery is currently a vivid area of research, both in pharmaceutical industry and in clinical environments. Practical and physiological reasons make the nasal mucosa an attractive surface area for drug delivery (Fig. 4). Applicatory procedures are simple, and the intranasal application with several concomitant benefits offers a real alternative to other application procedures (Table).

Table. Benefits of intranasal drug delivery

- Efficient absorption followed by rapid onset of drug effect

- Considerable bioavailability for many drugs

- Circumventing first-pass effect

- Convenient application (painless, no needle)

- Independent of ingestion

The mucosa-covered nasal cavities provide an absorptive surface of about 160 $\mathrm{cm}^{2}$. The considerable blood flow, responsible for breath warming, supports efficient systemic drug absorption. Hence gastrointestinal degradation and hepatic first-pass metabolism are circumvented, and systemic bioavailability is notably augmented. Thus, the onset of systemic effects of intranasally applied drugs often occurs earlier than after
Fig. 3. Penetration profile of triamcinolone acetonide (TACA), a middle potent corticosteroid, into stratum corneum after application of two different vehicles (acetone solution, ethanolic gel), and tapestripping after $0.5 \mathrm{~h}$. TACA amounts on each tape (horizontal bars) are correlated to the tape number (right scale) and to the depth of penetration into stratum corneum, displayed as percentage of the total stratum corneum thickness (left scale). This correlation is possible because the entire stratum corneum was stripped from each skin site; hence the total number of tapes represents $100 \%$ of the stratum corneum thickness. 


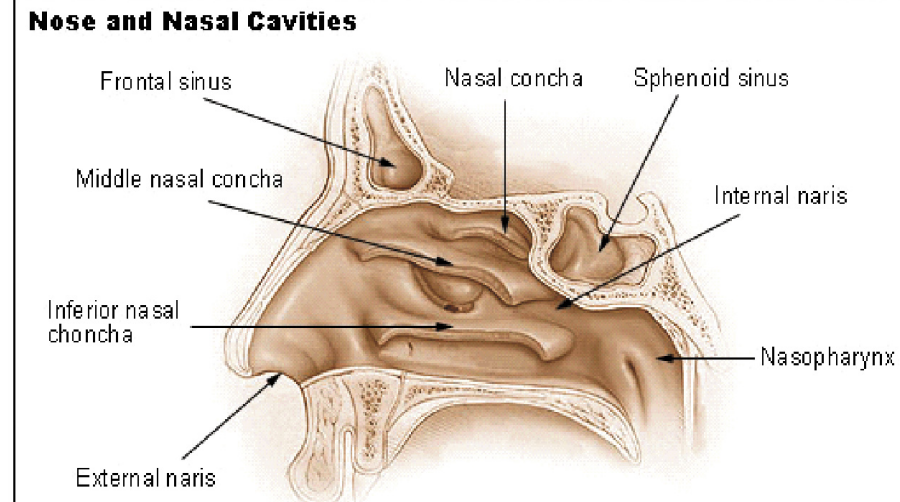

Fig. 4. Anatomy of the nasal cavities

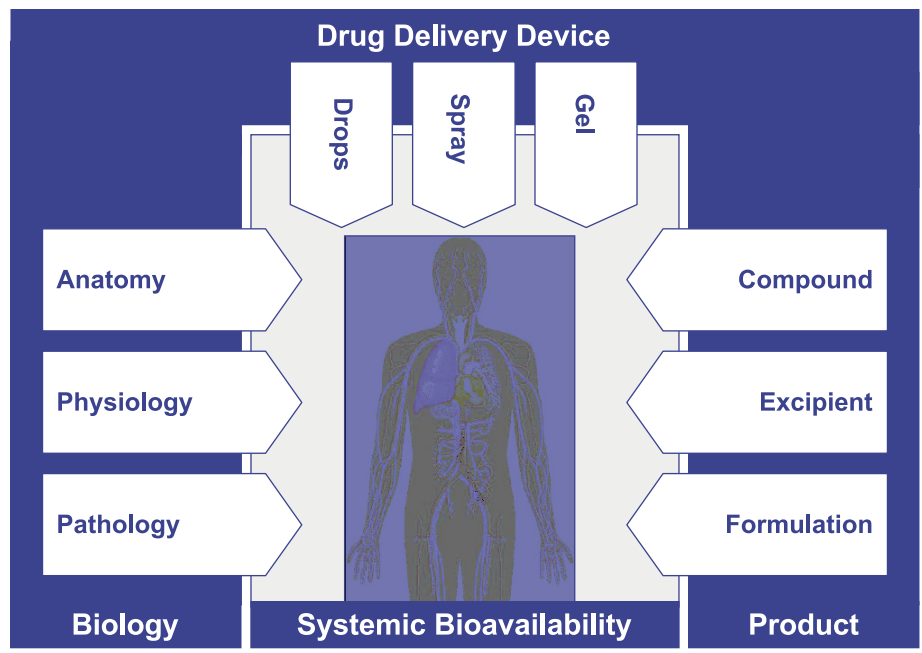

Fig. 5. Factors influencing the systemic bioavailability of nasally applied drugs

oral application. For a series of drugs, the plasma concentrations of intravenously and intranasally applied drugs are comparable [6]. However, the development of nasal formulations involves a careful tuning of several factors influencing application and drug absorption. These include the application device, the physiological environment and the formulation itself (Fig. 5).

\subsubsection{Clinical Use of Intranasal Midazolam Application}

In a clinical setting the intranasal administration of midazolam with a rapid and reliable onset of action has been of particular interest. In recent years clinicians relied on the intranasal application of the $i$.v. formulation of the branded midazolam product (Dormicum ${ }^{\circledR}$, Roche). However the product is not ideal for intranasal administration because of the low midazolam concentration and the acidity of the formulation $(\mathrm{pH} \approx 3)$. Furthermore intranasal irritation, discomfort in the throat area, as well as the bitter taste of the compound are common complaints following the intranasal administration [7].

This was the starting point of a mida-

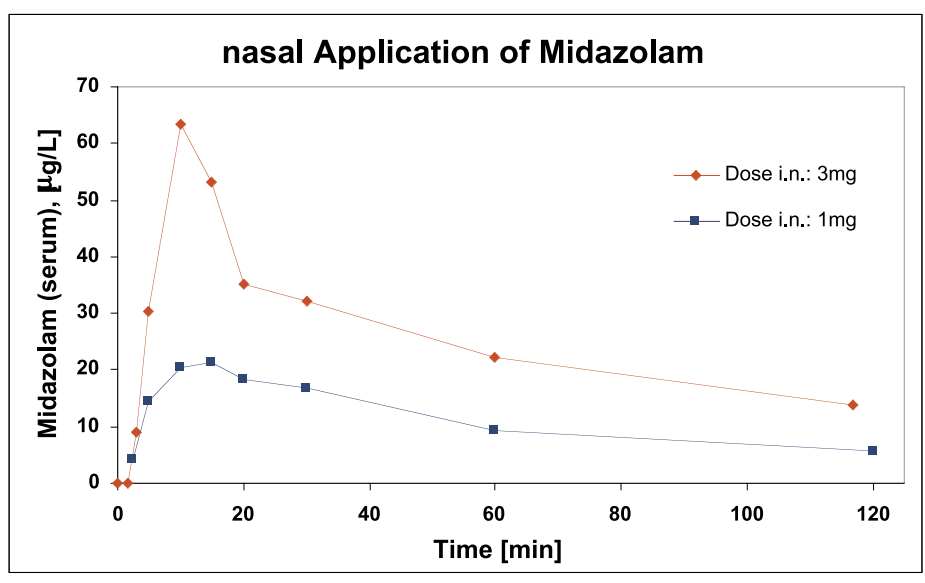

Fig. 6. Midazolam serum concentration after intranasal application

moderate to severe anxiety during magnetic resonance imaging (MRI), anxiolytic treatment is often provided by oral or intravenous application of midazolam prior to the clinical examination. Intranasal application of midazolam has been reported to be more suitable in anxiolysis avoiding sedation, and sustaining the capability of the patient for concurrent cooperation with the healthcare staff. Furthermore the rapid onset of the therapeutic effect is considered to be beneficial for the MRI workflow. Currently the anxiolytic potential of two nasal midazolam formulations is under clinical investigation in three radiology centres in Switzerland. Efficacy and patient comfort are being measured using visual analogue scales.

Received: January 16, 2006

zolam formulation development program at our institute. Based on extensive in vitro release studies using a variety of potential solubilisation enhancers, drug release retarders or permeation enhancers, formulations were selected for in vivo bioavailability studies.

In a preliminary investigation, the feasibility of an in vivo bioavailability study of nasally applied midazolam in human volunteers with the dose of $1 \mathrm{mg}$ (Dormicum ${ }^{\circledR}$, Roche, $5 \mathrm{mg} / \mathrm{ml})$ and $3 \mathrm{mg}(30 \mathrm{mg} / \mathrm{ml}$ formulation with solubilisation enhancer) was confirmed. Blood samples were drawn at multiple time points, and midazolam, as well as two metabolites, were quantified by a validated LC-MS method [8]. Maximal serum concentrations of $21 \mu \mathrm{g} / \mathrm{l}$ (dose: $1 \mathrm{mg}$ ) were reached within $15 \mathrm{~min}$, and 63 $\mu \mathrm{g} / \mathrm{l}$ (dose: $3 \mathrm{mg}$ ) within $10 \mathrm{~min}$ (Fig. 6). The delayed increase of $\alpha$-hydroxy- and 4-hydroxy-midazolam indicates that midazolam is absorbed nasally, circumventing first-pass metabolism. Currently the pharmacokinetic profiles of several midazolam formulations developed for the intranasal application are under investigation in human volunteers.

Since up to $30 \%$ of patients experience
[1] C. Surber, F.P. Schwarb, E.W. Smith, in 'Percutaneous Absorption: Drugs - Cosmetics - Mechanisms - Methodology', Eds. R.L. Bronaugh, H.I. Maibach, Taylor\&Francis, New York, 2005, p. 399 410, ISBN 1-57444-869-2.

[2] H. Oehman, A. Vahlquist, Acta Derm. Venereol. (Stockh) 1994, 74, 375-379.

[3] H.J. Weigmann, U. Lindemann, C. Antoniou, G.N. Tsikrikas, A.I. Stratigos, A. Katsambas, W. Sterry, J. Lademann, Skin Pharmacol. Physiol. 2003, 16, 217-227.

[4] C. Pellanda, E. Ottiker, C. Strub, T. Rufli, C. Surber, 2005, submitted.

[5] C. Pellanda, M. Weber, A.J. Bircher, C. Surber, Dermatology 2005, 211, 338340.

[6] T.R. Wolfe, T. Bernstone, J. Emerg. Nurs. 2004, 30, 141-147.

[7] H. Gudmundsdottir, J.F. Sigurjonsdottir, M. Masson, O. Fjalldal, E. Stefansson, T. Loftsson, Pharmazie 2001, 56, 963-966.

[8] F.E. Dussy, C. Hamberg, T.A. Briellmann, Int. J. Leg. Med. 2005, 12, 1-8. 